\title{
POSITIVE IMPACT OF PRESCRIBED PHYSICAL ACTIVITY ON SYMPTOMS OF SCHIZOPHRENIA: RANDOMIZED CLINICAL TRIAL
}

\author{
Dorde Curcic $^{1}$, Tamara Stojmenovic ${ }^{2}$, Slavica Djukic-Dejanovic ${ }^{1,3}$, Nenad Dikic ${ }^{2}$, MilicaVesic- \\ Vukasinovic $^{2}$, Nenad Radivojevic ${ }^{2}$, Marija Andjelkovic ${ }^{2}$, Milica Borovcanin ${ }^{3}$ \& Gorica Djokic $^{1}$ \\ ${ }^{I}$ Clinic for Mental Disorders "Dr Laza Lazarevic", Belgrade, Serbia \\ ${ }^{2}$ Sports Medicine Association of Serbia, Belgrade, Serbia \\ ${ }^{3}$ Faculty of Medical Science, University of Kragujevac, Kragujevac, Serbia
}

received: 18.10.2016;

revised: 27.3.2017;

accepted: 8.5 .2017

\section{SUMMARY}

Background: The purpose of this study was to examine functional capacity of cardio-respiratory system in patients with schizophrenia, and to evaluate the effects of 12 weeks prescribed physical activity on aerobic capacity and symptoms of schizophrenia.

Subjects and methods: Study involved 80 hospitalized patients with any of the subtypes of schizophrenia (42 men, 38 women). They were divided into two groups: exercise and control group, both with 40 patients. Maximal aerobic capacity (VO2 max) as an indicator of cardiovascular fitness has been obtained by cardiopulmonary stress test on a treadmill. Twelve weeks program of prescribed physical activity (45 minutes, four times per week) was made for every patient individually. Patients in exercise group practiced in training zone between 65 and 75\% of their maximum heart rate (HR). Target HR was controlled by Polar F4 monitors. Symptoms of schizophrenia were measured by using Positive and Negative Symptoms Scale (PANSS).

Results: Before the exercise program was introduced, measured VO2 max was significantly lower in patients with schizophrenia, than the expected average value in matched healthy subjects $(p<0.001)$. After twelve weeks, patients in exercise group showed a significant increase of VO2max ( $p=0.002)$, and significantly higher level of VO2max compared to the control group ( $p=0.000)$. Significant differences were also observed on PANSS general psychopathology subscale $(p=0.007)$ and on PANSS total score $(p=0.001)$. The pharmacotherapy and exercise had influence on PANSS general psychopathology ( $p=0.002)$ and PANSS total score $(p=0.001)$.

Conclusions: Individuals with schizophrenia have lower levels of aerobic capacity compared to general population. Prescribed physical activity significantly improves aerobic capacity in people with schizophrenia and it is effective in amelioration of some psychiatric symptoms. Prescribed physical activity could be an effective adjunctive treatment for patients with schizophrenia, not only for prevention and treatment of comorbidities, but also having an impact on symptoms of schizophrenia.

Key words: schizophrenia - physical activity - PANSS

\section{INTRODUCTION}

Individuals with serious mental illness are more likely to be sedentary than the general population (Faulkner et al. 2005). Consequently, they are at high risk to develop chronic medical conditions associated with inactivity. Physical inactivity is one of the most prevalent risk factors for obesity, metabolic syndrome and cardiovascular disease. High rates of cardiovascular disease have been observed in the schizophrenia population, translating into a life expectancy that is reduced by as much as $20-25 \%$ in comparison with mentally healthy controls (Laursen et al. 2012). The reasons are complex and include unhealthy lifestyles, poor diet, little exercise, and cigarette smoking (Brown et al. 1999, Strassnig et al. 2011), in addition to treatment with antipsychotic medications (Newcomer \& Hannekens 2007). The most effective medications for managing schizophrenia are mainly associated with weight gain, glucose dysregulation and diabetes (Beebe et al. 2005). The largest studies on population with schizophrenia confirmed the high prevalence of metabolic syndrome in this population, two times higher than in general population (McEvoy et al. 2005, Mitchell et al. 2013), and concluded that adjunct treatments are needed to mitigate such complications.

There are also indications that patients with schizophrenia have low levels of cardio-respiratory fitness (Strassnig et al. 2011). Low cardio-respiratory fitness has been recognized as an independent risk factor for all-cause mortality in adults (Lee et al. 2010). Strong evidence supports that increased cardio-respiratory fitness improves several parameters of physical health including body composition (Rippe \& Hess 1998), glucose control (Boulé et al. 2001), blood pressure (Kokkinos et al. 2009), and lipid profile (Kelley et al. 2005).

Physical activity is an important factor for the preservation of good general health and disease prevention. Exercise is inversely correlated with morbidity and mortality from several chronic diseases, including cardiovascular diseases (Harrington \& Gibson 2009). 
Aerobic exercise training in patients with the metabolic syndrome can be useful as a treatment strategy and provides support for a physical activity role in the prevention of chronic disease (Katzmarzyk et al. 2008).

In accordance with all of the above, physical activity definitely has a positive impact on the prevention of comorbidity of somatic diseases in patients with schizophrenia, as well as on their general health and life expectancy.

In the last ten years there is a growing recognition that exercise can enhance mental health. A large body of evidence indicates that exercise improves symptoms in depression and anxiety disorders (Dunn et al. 2005, Herring et al. 2010), but there is limited evidence to suggest that such benefits are also reported in population with schizophrenia. Most of the studies in this small body of literature have methodological limitations (notably small sample sizes and lack of randomization). Nevertheless, these studies consistently found physical exercise to be associated with reductions in depression and anxiety, and increased social interaction in persons with schizophrenia in both, inpatient and outpatient settings (Chamove 1986, Beebe et al. 2005, Acil et al. 2008).

Psychological changes such as social support, sense of autonomy, improved perceptions of competence, enhanced body image and self-efficacy are important for the improvement of some symptoms in schizophrenia (Mutrie \& Faulkner 2003). Exercise promotes physical self-worth and other important physical self-perceptions, such as improved body image. Physical activity is certainly a process that improves social relationships, while acting as a healthy way to the point of social interaction and networking.

Beside psychological changes, a large body of evidence indicates that locomotor activity can be beneficial in maintaining and even improving neural function following insult or disease (Pinilla et al. 2002, Luo et al. 2007). The most current hypothesis explaining the symptoms of schizophrenia indicate disturbed brain development and damaged connections between neurons. Aerobic exercise reinforces neural connections by increasing the number of dendrite connections between neurons, creating a denser network, which is more able to process and store information. The mechanisms for how exercise impacts neural function at the cellular and molecular levels are largely unexplored. No single mechanism has yet been found to adequately explain the diverse range of mental health effects possible through physical activity participation. Potential mechanisms are biochemical changes such as increased levels of neurotransmitters in central nervous system and neuroplasticity and other structural changes in brain (Achiron et al. 2008, Pajonk et al. 2010, Tzu \& Yu 2013, Falkai et al. 2013, Scheewe et al. 2013, Kimhy et al. 2015).

All these listed potential changes are interrelated and intertwined, and the final effect could be complex interactions between them. The aim of this study was to determine the patients' maximal aerobic capacityVO2max, and to examine how prescribed physical activity influence their cardio-respiratory fitness and symptoms of schizophrenia.

\section{SUBJECTS AND METHODS}

\section{Subjects}

This randomized control trial was performed in a Clinic for mental disorders "Dr Laza Lazarevic", Belgrade, Serbia. All experiments were conducted in accordance with the Declaration of Helsinki and approved by the Ethics Committee of the clinic. Written informed consent was obtained from all participants.

Sampling of the research consisted of 80 patients, between 25 and 65 years old, who were hospitalized upon the schizophrenia diagnoses (F20, any subtype) made according to International Classification of Diseases - Tenth Revision (ICD-10). Patients are involved in a program of exercise 10 days after hospitalization in the clinic.

During 18 months period, 146 potential participants were identified, 80 agreed to participate, and 66 declined. The most common reasons given for declining to participate were unwillingness to make a 12-week time commitment or physical problems, such as knees or back pain. The 80 persons agreeing to participate were randomly assigned to experimental (40 patients) or control groups (40 patients) by using simple randomization method. Exclusion criteria were cardiovascular diseases or acute infectious diseases. Patients had no primary diagnosis of alcohol or substance abuse. Antipsychotic medication was recorded for each patient and converted into a daily equivalent dosage of chlorpromazine, according to Gardner and colleagues (2010).

\section{Methods}

A multidisciplinary approach was applied to the study, as well as individual approach to each patient. Subjects underwent a physical examination, baseline electrocardiogram (ECG) and anthropometric measurements (body height and weight, body fat percentage) in the premises of Sports Medicine Association of Serbia (SMAS). Height was measured by Seca stadiometer 222, Hamburg, to the nearest $0.1 \mathrm{~cm}$. A high caliber scale (Tanita BC-418MA, Tokio), was used to measure body weight and body fat percentage (FAT\%). VO2 max is widely accepted as the single best measure of cardiovascular fitness and maximal aerobic capacity.

Familiarization to the treadmill, warm-up, and the VO2 max ramp-procedure have been described in detail previously to every patient. Subjects were then equipped with a facemask and ECG device (Quarck T 12x, Wireless 12-lead ECG) before starting the cardiorespiratory test on treadmill. Heart rate was monitored by COSMED Wireless HR Monitor and by ECG. 
Volitional exhaustion, RR over 1.1 or plateau of VO2, patient request, electrocardiographic or hemodynamic abnormalities were the reasons for test termination. All tests were performed by trained sport medicine specialist of SMAS. Twelve weeks exercise program (45 minutes, four times per week) was designed for each patient according to his age and skill level. Prescribed exercised zone was $65-75 \%$ max heart rate, determined by sport exercise specialists and according to previous studies (Meyer \& Broocks 2000, Gold et al. 2003, Jerome et al. 2009).

Before starting program, our patients were educated about the importance of physical activity and its benefits for health, than about the target heart rate for their age, muscle-stretching warm-up and cool-down exercises. The program consisted of five minutes of warm-up stretches, than experimental participants had a fast walk or jogging (depending of their heart rate) for 30 minutes. Patients were trained outside in the landscaped backyard of clinic. During walking and running, they crossed between 2 and $4 \mathrm{~km}$ in one training. At the end of session they had 10 minutes of stretching. Exercise therapy was supervised by a fitness trainer. Control group did not participate in any exercises during this time period, but they continued treatment with the standard pharmacological therapy.

\section{Instrument}

Symptoms of schizophrenia were measured by using Positive and Negative Symptoms Scale (PANSS). The PANSS (Kay et al. 1987) was designed to measure severity of psychopathology in adults with schizophrenia along positive, negative, and general dimensions. The PANSS includes 30 items scored on a scale from 1 to 7 ; items are summed to determine the subscale and total scores, with higher scores indicating greater severity of symptoms. PANSS was done after the patients signed informative consent. The next day, patients started with a program of physical activity. The day after completing the program, PANSS and treadmill test were repeated. Testing on the treadmill was done by the doctor who had nothing to do with the treatment of the patients, nor knew which patients were included in the study. Scoring at PANSS did psychiatrist who was not treating the patients included in the study.

\section{Statistical analyses}

Statistical analyses were performed with the software IBM SPSS, version 20.0. All data were assessed for normality (one-sample Kolmogorov-Smirnov test). Descriptive statistics of the groups data are given as mean \pm SD. Significant changes between exercise and control group in $\mathrm{VO} 2 \mathrm{max}$ were analyzed by using ttest. Significant changes within each group during 12 weeks period were analyzed by using t- test for paired samples. A comparison between the group changes and influence of pharmacotherapy and exercise effects on measured parameters of schizophrenia was performed by general linear model two-way analysis of variance (ANOVA). A p-value $<0.05$ was considered statistically significant.

\section{RESULTS}

The gender distribution in the final sample was 42 $(52.5 \%)$ men and 38 (47.5\%) women. The experimental group consisted of 23 men and 17 women, while in the control group there were 19 men and 21 women. Patients were treated with antipsychotics, as shown in Table 1 . They did not receive any antidepressants nor beta-blockers. Other demographic and clinical characteristics of participants are presented in Table 1.

Table 1. Demographic and clinical characteristics of patients with schizophrenia $(\mathrm{N}=80)$

\begin{tabular}{|c|c|c|c|c|c|c|c|}
\hline & \multicolumn{3}{|c|}{ Exercise group } & \multicolumn{4}{|c|}{ Control group } \\
\hline & Men & Women & Total & Men & Women & Total & $\begin{array}{l}\mathrm{P} \text { exercise vs } \\
\text { control total }\end{array}$ \\
\hline Number of patients & 23 & 17 & 40 & 19 & 21 & 40 & \\
\hline Age (years) & $40.04 \pm 9.30$ & $39.82 \pm 10.09$ & $39.95 \pm 9.51$ & $40.16 \pm 9.07$ & $43.19 \pm 9.77$ & $41.75 \pm 9.45$ & 0.398 \\
\hline Duration of illness (years) & $13.00 \pm 7.53$ & $11.44 \pm 7.70$ & $12.52 \pm 7.62$ & $11.40 \pm 7.11$ & $10.31 \pm 7.32$ & $11.14 \pm 7.21$ & 0.227 \\
\hline \multicolumn{8}{|l|}{ Antipsychotic medicine } \\
\hline FGA & 6 & 4 & 10 & 7 & 5 & 12 & \\
\hline SGA & 11 & 10 & 21 & 8 & 8 & 16 & \\
\hline Antipsychotic polypharmacy & 6 & 3 & 9 & 4 & 8 & 12 & \\
\hline Antipsychotic dosage $(\mathrm{mg} * \mathrm{~d}-1)^{\mathrm{a}}$ & $733.5 \pm 327.1$ & $628.8 \pm 345.2$ & $689.0 \pm 310.7$ & $771.6 \pm 390.2$ & $637.7 \pm 287.9$ & $701.3 \pm 301.5$ & 0.527 \\
\hline BMI $\left(\mathrm{kg} / \mathrm{m}^{2}\right)$ & $25.96 \pm 3.10$ & $27.62 \pm 2.53$ & $26.67 \pm 2.96$ & $26.24 \pm 1.69$ & $26.56 \pm 2.66$ & $26.41 \pm 2.23$ & 0.662 \\
\hline$\%$ fat & $23.91 \pm 3.89$ & $34.52 \pm 5.06$ & $28.42 \pm 6.87$ & $25.03 \pm 3.62$ & $33.94 \pm 5.48$ & $29.71 \pm 6.46$ & 0.347 \\
\hline
\end{tabular}

Table 2. VO2 max levels before and after twelve weeks program

\begin{tabular}{lcccccc}
\hline & \multicolumn{3}{c}{ Baseline } & \multicolumn{3}{c}{ Follow-up } \\
VO2 $\max (\mathrm{ml} / \mathrm{kg} / \mathrm{min})$ & Men & Women & Total & Men & Women & Total \\
\hline Exercise group & $21.68 \pm 2.53$ & $18.81 \pm 1.62$ & $20.52 \pm 2.52$ & $24.28 \pm 2.47$ & $21.46 \pm 2.17$ & $23.08 \pm 2.71$ \\
Control group & $22.15 \pm 2.08$ & $18.95 \pm 1.51$ & $20.47 \pm 2.40$ & $22.31 \pm 2.30$ & $19.15 \pm 1.70$ & $20.65 \pm 2.55$ \\
\hline
\end{tabular}


Table 3. PANSS scores before and after exercise program

\begin{tabular}{lcccccc}
\hline \multirow{2}{*}{ VO2 max (m1/kg/min) } & \multicolumn{3}{c}{ Baseline } & & \multicolumn{3}{c}{ Follow-up } \\
& Exercise group & Control group & $\mathrm{p}$ & Exercise group & Control group & $\mathrm{p}$ \\
\hline PANSS positive & $18.33 \pm 6.08$ & $18.83 \pm 7.03$ & 0.836 & $14.13 \pm 3.28$ & $15.70 \pm 5.38$ & 0.364 \\
PANSS negative & $22.60 \pm 7.09$ & $21.23 \pm 9.10$ & 0.381 & $16.75 \pm 5.51$ & $17.95 \pm 6.63$ & 0.407 \\
PANSS general psychopathology & $49.60 \pm 10.13$ & $51.25 \pm 8.61$ & 0.435 & $44.08 \pm 8.75$ & $47.95 \pm 7.15$ & 0.007 \\
PANSS Overall & $89.75 \pm 11.22$ & $92.35 \pm 12.48$ & 0.330 & $74.48 \pm 10.86$ & $83.03 \pm 10.50$ & 0.001 \\
\hline
\end{tabular}

Table 4. Pharmacotherapy and exercise effects on symptoms of schizophrenia

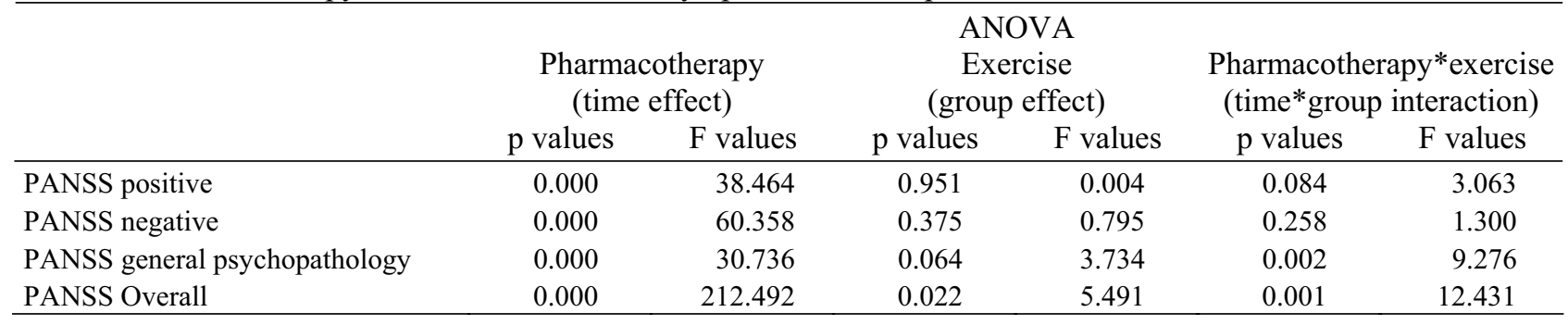

All patients from both groups did cardiopulmonary stress test on a treadmill before they start exercise. Mean value of maximal aerobic capacity was $21.78 \pm 2.34 \mathrm{ml} / \mathrm{kg} / \mathrm{min}$ for men and $17.89 \pm 1.64$ $\mathrm{ml} / \mathrm{kg} / \mathrm{min}$ for women. No significant pre-exercise differences between exercise and control groups were found for VO2 $\max$ in both genders (men: $\mathrm{t}$-value $=-0.493$, $\mathrm{df}=40 ; \quad \mathrm{p}=0.625$; women: $\mathrm{t}$-value $=0.275, \mathrm{df}=36$, $\mathrm{p}=0.785)$. According to World Health Organization classification of level of aerobic capacity by gender and age, $97.5 \%$ patients were in the group "very low", and only 2 patients $(2.5 \%)$ had mark "low". Compared to average expecting values for general population for the same age (Zivanic et al. 1999), patients participating in the study had significant lower level of VO2 max. Average expected value for the general population of the same age is $29.97 \pm 4.24 \mathrm{ml} / \mathrm{kg} / \mathrm{min}$, and VO2 max of our patients was $20.50 \pm 2.45$ (t-value $=-26.241$, $\mathrm{df}=79$, $\mathrm{p}<0.001)$.

After 12 weeks, patients in exercise group showed significantly increases of $\mathrm{VO} 2 \max (\mathrm{t}$-value $=-3.711$, $\mathrm{df}=16, \mathrm{p}<0.001)$. In both genders difference was statistically significant: maximal aerobic capacity increased from 21.68 to $24.28 \mathrm{ml} / \mathrm{kg} / \mathrm{min}$ among males ( $\mathrm{t}$-value $=-7.770, \mathrm{df}=22, \mathrm{p}<0.001$ ), and in female sample from 18.81 to $21.46 \mathrm{ml} / \mathrm{kg} / \mathrm{min}$ among 17 females ( $\mathrm{t}$-value $=-3.711, \mathrm{df}=16, \mathrm{p}=0.002$ ) (Table 2). In control group, changes in VO2 max before and after 12 weeks period were minimal and not statistically significant ( $\mathrm{t}$-value $=-1.384, \mathrm{df}=18, \mathrm{p}=0.183$ for men, $\mathrm{t}$-value $=-1.356$, $\mathrm{df}=20, \mathrm{p}=0.190$ for women and $\mathrm{t}$-value $=-1.939, \mathrm{df}=39$, $\mathrm{p}=0.060$ for all patients in control group). Intergroup comparison at the end of study showed that VO2 max values were much higher in exercise group $(23.08 \pm 2.71$ $\mathrm{ml} / \mathrm{kg} / \mathrm{min})$ then in control group $(20.65 \pm 2.55$ $\mathrm{ml} / \mathrm{kg} / \mathrm{min})(\mathrm{t}$-value $=5.001, \mathrm{df}=38, \mathrm{p}<0.001)$.

PANSS Positive, Negative and General Psychopathology subscores and PANSS total score, before and after exercise, for both groups are shown in Table 3. At baseline, before experimental period, no significant differences were found on subscales, as well as on PANSS total score $(\mathrm{t}$-value $=0.980, \mathrm{df}=78, \mathrm{p}=0.330)$. After exercise period, statistically significant differences between groups were found on PANSS general psychopathology subscale ( $\mathrm{t}$-value $=-2.847, \mathrm{df}=78, \mathrm{p}=0.007$ ) and PANSS total ( $\mathrm{t}$-value $=-3.630, \mathrm{df}=78, \mathrm{p}=0.001$ ) in terms of lower values in the group who had physical activity.

Between the group changes and influence of pharmacotherapy and exercise effects on measured parameters of schizophrenia are shown in Table 4. ANOVA revealed a significant main effect of pharmacotherapy (time) on symptoms of schizophrenia $(p<0.001$ in all subscores and in PANSS total score). Furthermore, ANOVA analysis also showed a significant time and group interaction effect on PANSS general psychopathology $(p=0.002)$ subscale and PANSS total score $(\mathrm{p}=0.001)$. Interaction effect was not statistically significant in PANSS positive and PANSS negative subscores.

\section{DISCUSSION}

Individuals with schizophrenia have unhealthy lifestyle with little or no exercise. They score less on physical activity and fitness measures than persons with no mental illness and persons with other mental illnesses (Chamove 1986). Therefore, our results showing that individuals with schizophrenia had significantly lower levels of aerobic capacity compared to general population are in line with expectations. It is important to note that level of cardio-respiratory fitness in our patients was between 30-40\% lower compared with average expecting values for general population. Also $97.5 \%$ of them were in 'low fitness category'. Low cardio-respiratory fitness and obesity risks associated with atypical antipsychotic medications led psychiatrists to a greater awareness of the importance of reducing risk factors through physical activity in this population (Heald 2010). 
We showed that prescribed, dosed physical activity improves aerobic capacity in our patients. These results are congruent with the findings of Wilmore et al. (2001), which showed that a previously sedentary person may experience a highly relevant increase in VO2 max after 8 weeks of structured training (as much as a $25 \%$ ). Low cardio-respiratory fitness is an eminently modifiable risk factor, as it closely correlates with habitual physical activity. We have shown the same progress in VO2 max, but in a specific population of patients with schizophrenia. Exercise is generally a safe physical intervention and is relatively easy and inexpensive to implement. The contraindications are rare and not specific to people with mental disorders. The American College of Sports Medicine recommends that most adults engage in a regular exercise regime in order to maintain health and wellbeing, suggesting that higher activity levels are associated with greater health outcomes (American College of Sports Medicine 2007). When we consider the fact that an increasing number of patients suffering from schizophrenia use atypical antipsychotics and that they have a sedentary lifestyle, it is clear that they are at high risk of metabolic syndrome and subsequent serious cardiovascular complications. Physical activity is excellent therapy for both, preventing the onset of metabolic syndrome and cardiovascular diseases, in situations where the syndrome has already reported.

Beebe with colleagues (2005) did 6-minute walking distance test with 10 patients with schizophrenia. They made treadmill exercise program, three times a week, for 16 weeks. After conclusion of the exercise program, PANSS total score for these patients was 12 point lower compared with control group. In our study, significant improvement in symptoms was also observed, but in a larger sample. Acil and colleagues (2008) applied an aerobic exercise program in the periods of 10 weeks, 3 days in a week. They found decrease in positive symptoms and decrease in negative symptoms of the patients by means of physical exercise. We obtained the same results in a larger number of patients, but also we had an individualized approach, dosed physical activity, which is much more comfortable and easier for the patient and reduces the risk of adverse events. The motivation and cooperation are certainly the most important aspect of any therapy. Faulkner (2005) concludes that exercise may alleviate symptoms of schizophrenia such as depression, low self-esteem and social withdrawal. Our results confirm this claim, which is clearly seen in the PANSS general psychopathology subscale. Gorczynski and Faulkner (2010) in Cochrane review conclude that exercise can have healthful effects on both the physical and mental health and well-being of individuals with schizophrenia. They conclude that larger randomized studies are required before any definitive conclusions can be drawn. Finally, Soundy and colleagues (2015) in systematic review conclude that sport participation may result in reduced psychiatric symptoms in patients with schizophrenia.

Physical activity cannot replace traditional treatments of serious psychiatric illnesses, but could play an important part of the treatment process (Kimhy 2014). We reported reductions in PANSS general psychopathology symptoms post-exercise. Our results of reductions in psychiatric symptoms as measured by the PANSS are congruent with the findings of Faulkner (2005) as well as Pelham and colleagues (1993) that aerobic exercise significantly reduced depression and anxiety in persons with schizophrenia. In each subscale of PANSS we made greater shift in the group that practiced compared to group with just "traditional" treatment. The difference was statistically significant in subscale general psychopathology, but it is undisputed that there are differences in each subscale. These small differences, which may not be statistically significant, can be very important in the clinical sense. Finally, the difference in PANSS total score between the control and experimental group was highly statistically significant.

For many people, physical activity and exercise is an important source of happiness in their lives. Regardless of its therapeutic properties, there are no reasons for people with schizophrenia not to take pleasure in the same type of activities. No doubt, there are many difficulties of conducting methodologically studies of exercise as an adjunct treatment for schizophrenia. Schizophrenia with wide range of symptoms includes wide variability in baseline functioning, motivation, pharmacologic treatments, smoking habits, which makes drawing comparisons across populations problematic. Responses to exercise and preferred modalities are highly individualized. Therefore, good relationship with the patients and motivation are important factors. We have tried to show the impact of physical activity on patients with schizophrenia, with no intention to discuss doses of drugs taken by patients during treatment. Finally, differences across inpatient and outpatient; young and older patients hamper generalization.

Future studies should follow patients over a longer time period, their quality of life, somatic complications that they will have, or will be potentially prevent by physical activity. Additional studies are needed to systematically examine the interactions of medications with the effects of exercise, as well as any differences in outcomes based on type, frequency, and duration of exercise sessions to examine responses to different exercise dosages. Further studies are needed to identify the most effective exercise interventions and the most feasible delivery modalities for inpatient, outpatient, younger or older patients. Psychiatrists and general practitioners need to be consistent and persistent in encouraging patients to be physically active and continue to highlight the benefits of exercise to physical as well as mental health. 


\section{CONCLUSIONS}

Physical inactivity is, according to the WHO, the fourth leading risk factor for global mortality following hypertension, tobacco use and high blood glucose. Individuals with schizophrenia have unhealthy lifestyle with little or no exercise. Consequently, level of their cardio-respiratory fitness is lower than in general population. In our patients VO2 max was between 30$40 \%$ lower compared with average expecting values for general population. The vast majority was in 'low fitness category'. Low cardio-respiratory fitness and obesity risks associated with atypical antipsychotic medications, are a great risks for the occurrence of somatic complications in this group of patients and increased mortality. Therefore it is crucial to consider reducing risk factors where possible. Prescribed physical activity significantly improves aerobic capacity in people with schizophrenia, and it is a safe intervention and relatively easy and inexpensive to implement.

Undoubtedly pharmacological treatment (antipsychotics) is a basic for patients with schizophrenia, and physical activity could be adjunct therapy and part of integrative approach and treatment. In addition to being undeniably reduces the risk of co morbidity, it is effective for decreasing some psychiatric symptoms. Prescribed physical exercise could be an effective adjunct treatment for patients with schizophrenia, not only for the prevention and treatment of co morbidities, but also to reduce and mitigate symptoms of schizophrenia themselves.

\section{Acknowledgements: None.}

Conflict of interest: None to declare.

\section{Contribution of individual authors:}

Dorde Curcic: Concept, design, resources, materials data collection, data processing, analysis/interpretation, literature search and analyses, writing manuscript, supervision;

Tamara Stojmenovic: Design of study, resources, writing manuscript, materials data collection, critical review;

Slavica Djukic-Dejanovic: Design of study, interpretation of data, first draft, critical review;

Nenad Dikic: Design of study, interpretation of data, first draft, critical review;

Milica Vesic-Vukasinovic: Data processing, analysis/ interpretation, literature search;

Nenad Radivojevic: Supervision, materials, critical review;

Marija Andjelkovic: Data processing, analysis/interpretation, literature search;

Milica Borovcanin: Materials, data collection, data interpretation, first draft;

Gorica Djokic: Design study, resources, materials, data collection, data interpretation.

\section{References}

1. Acil AA, Dogan $S$ \& Dogan O: The effects of physical exercises to mental state and quality of life in patients with schizophrenia. J Psychiat Ment Health Nurs 2008; 15:808-15.

2. Achiron A \& Kalron A: Physical activity: Positive impact on brain plasticity. Harefuah 2008; 147:252-5.

3. American College of Sports Medicine: Guidelines of Exercise Testing and Prescription, 7th edition. Williams \& Wilkins, Baltimore, 2005.

4. American Psychiatric Association: Diagnostic and statistical manual of mental disorders (4th ed., text rev.). Author, Washington DC, 2000.

5. Beebe LH, Tian L, Morris N, Goodwin A, Allen SS \& Kuldau J: Effects of exercise on mental and physical health parameters of persons with schizophrenia. Issues in Ment Health Nurs 2005; 26:661-76.

6. Boule NG, Hadad E, Kenny GP, Wells GA \& Sigal RY: Effects of exercise on glycemic control and body mass in type 2 Diabetes mellitus: A meta analyze of controlled clinical trials. JAMA 2001; 286:1218-27.

7. Brown $S$, Birtwistle $J$, Roe $L$ \& Thompson $C$ : The unhealthy lifestyle of people with schizophrenia. Psychol Med 1999; 29:697-701.

8. Chamove AS: Positive short-term effects of activity on behavior in chronic schizophrenic patients. $\mathrm{Br} J$ Clin Psychol 1986; 25:125-33.

9. Dunn AL, Trivedi MH, Kampert JB, Clark CG \& Chambliss HO: Exercise treatment for depression: Efficacy and dose response. Am J Prev Med 2005; 28:1-8.

10. Falkai P, Malchow B, Wobrock T, Gruber O, Schmitt A \& Honer WG: The effect of aerobic exercise on cortical architecture in patients with chronic schizophrenia: a randomized controlled MRI study. Eur Arch Psychiatr Clin Neurosci 2013; 263:469-73.

11. Faulkner $G$ \& Taylor AH: Exercise, Health and Mental Health: Emerging relationships between physical activity and psychological well-being. 1st Edition. Routledge, London, 2005.

12. Fogarty M: Exploring the benefits of an exercise program for people with schizophrenia: A qualitative study. Issues in Ment Health Nurs 2005; 26:341-51.

13. Gardner DM, Murphy AL, O'Donnell H, Centorrino $F \&$ Baldessarini RJ: International consensus study of antipsychotic dosing. Am J Psychiatry 2010; 167:686-93.

14. Gold SM, Schulz KH, Hartmann S, Mladek M, Lang UE, Hellweg $R$ et al.: Basal serum levels and reactivity of nerve growth factor and brain-derived neurotrophic factor to standardized acute exercise in multiple sclerosis and controls. J Neuroimmunol 2003; 138:99-105.

15. Gorczynski $P$ \& Faulkner G: Exercise therapy for schizophrenia. Cochrane Database Syst Rev 2010; 5: doi: 10.1002/14651858.CD004412.pub2.

16. Harrington M, Gibson $S$ \& Cottrell RC: A review and meta-analysis of the effect of weight loss on all-cause mortality risk. Nutr Res Rev 2009; 22:93-108.

17. Heald A: Physical health in schizophrenia: A challenge for antipsychotic therapy. Eur Psychiatry 2010; 25: 6-11.

18. Herring MP, O'Connor PJ \& Dishman RK: The effect of exercise training on anxiety symptoms among patients: A systematic review. Arch Intern Med 2010; 170:321-31.

19. Jerome GJ, Young DR, Dalcin A, Charleston J, Anthony $C$, Hayes $J$ et al.: Physical activity levels of persons with 
mental illness attending psychiatric rehabilitation programs. Schizophr Res 2009; 108:252-7.

20. Kay SR, Fiszbein A \& Opler LA: The Positive and Negative Syndrome Scale for schizophrenia. Schizophr Bull 1987; 13:261-76.

21. Katzmarzyk PT, Leon AS, Wilmore JH, Skinner JS, Rao $D C$, Rakinen $T$ et al.: Targeting the Metabolic Syndrome with Exercise: Evidence from the HERITAGE Family Study Med \& Sci in Sports \& Exerc 2008; 35:1703-9.

22. Kelley GA, Kelley KS \& Vu Tran Z: Exercise, Lipids, and Lipoproteins in Older Adults: A Meta-Analysis. Prev Cardiol 2005; 8:206-14.

23. Kimhy D, Vakhrusheva J, Bartels MN, Armstrong HF, Ballon JS, Khan Set al.: Aerobic fitness and body mass index in individuals with schizophrenia: Implications for neurocognition and daily functioning. Psychiatry Res 2014; 220:784-91.

24. Kimhy D, Vakhrusheva J, Bartels MN, Armstrong HF, Ballon JS, Khan $S$ et al.: The impact of aerobic exercise on brain-derived neurotrophic factor and neurocognition in individuals with schizophrenia: A single-blind, randomized clinical trial, Schizophr Bull 2015; 41: 859-68.

25. Kokkinos $P$, Manolis A, Pittaras A, Doumas M, Giannelou, Demosthenes B et al.: Exercise capacity and mortality in hypertensive men with and without additional risk factors. Hypertension 2009; 53: 494-9.

26. Laursen TM, Munk-Olsen T\& Vestergaard M: Life expectancy and cardiovascular mortality in persons with schizophrenia. Curr Opin Psychiatry 2012; 25: 83-8.

27. Luo CX, Jiang J, Zhou QG, Zhu XJ, Wang W\& Zhang ZJ: Voluntary exercise-induced neurogenesis in the postischemic dentate gyrus is associated with spatial memory recovery from stroke. Jour of Neurosci Res 2007; 85:1637-46.

28. Lee D, Artero EG, Sui G \& Blair SN: Mortality trends in the general population: the importance of cardio respiratory fitness. J Psychopharmacol. 2010; 24:27-35.

29. Meyer $T \&$ Broocks A: Therapeutic impact of exercise on psychiatric diseases: Guidelines for exercise testing and prescription. Sports Med 2000; 30: 269-79.

30. McEvoy JP, Meyer JM, Goff DC, Nasrallah HA, Davis $S M$, Sullivan $L$ et al.: Prevalence of the metabolic syndrome in patients with schizophrenia: Baseline results from the Clinical Antipsychotic Trials of Intervention Effectiveness (CATIE) schizophrenia trial and comparison with national estimates from NHANES III. Schizophr Res 2005; 80:19-32.

31. Mitchell AJ, Vancampfort D, Sweers $K$, van Winkel R, Yu $W \&$ de Hert M: Prevalence of metabolic syndrome and metabolic abnormalities in schizophrenia and related disorders-asystematic review and meta-analysis. Schizophr Bull 2013; 39:306-18.

32. Mutrie $N$ \& Faulkner G: Physical activity and mental health. In: Everett T, Donaghy $M$ \& Fever $S$ (eds):
Physiotherapy and Occupational Therapy in Mental Health: An evidence based approach, 82-97. Routledge, 2003.

33. Newcomer $J$ \& Hannekens CH: Severe mental illness and risk of cardiovascular disease editorial. J Am Med Assoc 2007; 15:1794-6.

34. Pajonk FG, Wobrock T, Gruber O, Scherk H, Berner D, Kaizl I et al.: Hippocampal plasticity in response to exercise in schizophrenia. Arch Gen Psychiatry 2010; 67:133-43.

35. Pelham TW, Campagna PD, Ritvo $P G \&$ Birnie WA: The effects of exercise therapy on clients in a psychiatric rehabilitation program. Psychosocial Reh J 1993; 16:75-84.

36. Pinilla FG, Ying $Z$, Roy RR, Molteni $R$ \& Edgerton VR: Voluntary Exercise Induces a BDNF-Mediated Mechanism That Promotes Neuroplasticity. Jour Neurophys 2002; 88:2187-95.

37. Rippe JM \& Hess S: The role of physical activity in prevention and management of obesity. J Am Diet Assoc 1998; 98:531-8.

38. Scheewe TW, van Haren NE \& Sarkisyan G: Exercise therapy, cardio respiratory fitness and their effect on brain volumes: a randomised controlled trial in patients with schizophrenia and healthy controls. Eur Neuropsychopharmacol 2013; 23:675-85.

39. Soundy A, Roskell C, Stubbs B, Probst M \& Vancampfort $D$ : Investigating the benefits of sport participation for individuals with schizophrenia: A systematic review. Psychiatr Danub 2015; 27: 2-13.

40. Strassnig M, Jaspreet S, Brar C\& Ganguli R: Low cardiorespiratory fitness and physical functional capacity in obese patients with schizophrenia. Schizophr Res 2011; 126:103-9.

41. Tzu-Wei L \& Yu-Min K: Exercise Benefits Brain Function: The Monoamine Connection. Brain Sci 2013; 3: 39-53.

42. Vancampfort D, Probst M, Helvik Skjaerven L, CatalánMatamoros D, Lundvik-Gyllensten A, Gómez-Conesa A et al.: Systematic review of the benefits of physical therapy within a multidisciplinary care approach for people with schizophrenia. Phys Ther 2012; 92: 11-23.

43. Vancampfort D, Probst $M$, Sweers $K$, Maurissen $K$, Knapen $J$ \& de Hert M: Relationships between obesity, functional exercise capacity, physical activity participation and physical self-perception in people with schizophrenia. Acta Psychiatr Scand 2011; 123: 423-30.

44. Wilmore JH, Stanforth PR, Gagnon T, Rice S, Mandel AS, Leon DC et al.: Heart rate and blood pressure changes with endurance training: The HERITAGE Family Study. Med Sci Sports Exerc 2001; 33: 107-16.

45. WHO: The ICD-10 classification of mental and behavioural disorders - diagnostic criteria for research. World Health Organization, Geneva, 1993.

46. Zivanic S, Zivotic-Vanovic M, Mijic R \& Dragojevic R: Aerobna sposobnost. Zelnid, Belgrade, 1999.

Correspondence:

Dorde Curcic, MD, PhD

Clinic for Mental Disorders "Dr Laza Lazarevic"

Pavla Bakica 6, 11000 Belgrade, Serbia

E-mail:dzordzic@yahoo.com 